\title{
Mise en place orthodontico-chirurgicale des canines incluses : moyens de prévisions des échecs
}

\section{RÉSUMÉ}

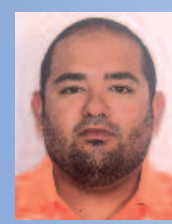

Mohamed Fadel ZEROUAOUI

Résidant en orthopédie dento-faciale à la faculté de médecine dentaire de Rabat, Université Mohamed V-Soussi,

Faculté de médecine dentaire,

Les instituts,

PB 6212 Rabat, Maroc.

Loubna BAHIJE

Professeur assistante

en orthopédie dento-faciale,

Faculté de médecine dentaire,

Rabat, Maroc.

Fatima ZAOUI

Professeur d'enseignement supérieur en orthopédie dento-faciale,

Chef de service d'orthopédie dento-faciale,

Faculté de médecine dentaire,

Rabat, Maroc.

\section{Wiam RERHRHAYE}

Professeur agrégée

en orthopédie dento-faciale,

Faculté de médecine dentaire,

Rabat, Maroc.

Chaque dent possède son importance dans la cavité buccale et par conséquent, son absence pourrait entraîner un déséquilibre et avoir une répercussion sur la santé des structures buccales environnantes. Devant des situations d'inclusion dentaire, des examens cliniques et radiologiques sont nécessaires pour poser le diagnostic positif et le pronostic.

Ainsi, plusieurs attitudes thérapeutiques sont possibles allant de l'abstention aux extractions chirurgicales par alvéolectomie. Toutefois, la thérapeutique la plus adoptée et la plus souhaitée est la mise en place orthodontico-chirurgicale.

Notre travail se propose de faire le point sur les moyens de prévision des échecs que peut rencontrer cette thérapeutique.

canine incluse

traction orthodontico-chirurgicale

- échecs
AOS 2012;260:313-325

DOI: $10.1051 / a 0 s / 2012403$

(C) EDP Sciences 


\section{Introduction}

$>$

L'inclusion des canines est, après celle des troisièmes molaires, la plus fréquemment rencontrée dans une fourchette de $0,9 \%$ à $2,2 \%$ d'après Ericson et Kurol. La canine inférieure a une fréquence $d$ 'inclusion 10 à 20 fois moindre que la canine supérieure, de l'ordre de 0,35\% selon Dachi et Howell ainsi que Begg [1-4].

La littérature rapporte une incidence de $10 \%$ de cas de canines en position très apicale. Elle est le plus souvent unilatérale, plus fréquente à droite qu'à gauche, et le maxillaire est de loin plus concerné que la mandibule par ce phénomène [1-8].

L'étiologie de l'inclusion canine peut être liée à $[2,3,5,8,9,10,11]$ :

- des facteurs environnementaux : absence de guidage liée à l'agénésie de l'incisive latérale, obstruction du chemin d'éruption

\section{Moyens de prévision}

La traction orthodontico-chirurgicale de la canine peut se heurter à des difficultés qui constituent un échec thérapeutique. Le praticien dispose de différents moyens de prévision qui commencent dès l'examen clinique et passent par le respect des étapes thérapeutiques.

\section{Moyens cliniques \\ et radiologiques}

\section{$>$ Moyens cliniques}

Ils sont basés sur l'inspection et la palpation qui permettent de pressentir la voie d'abord (dents surnuméraires, odontomes, mésiodens) ;

- des facteurs généraux : génétiques, endocriniens, nutritionnels et maladies infectieuses ;

- des facteurs iatrogènes : rétractions orthodontiques trop précoces, et réalisées de manière non critique, du bloc incisif avant l'éruption des canines.

Toute prise en charge orthodontico-chirurgicale de cette inclusion doit se baser sur une localisation la plus précise possible de la canine incluse dans les trois plans de l'espace. De même, il est primordial de respecter toutes les étapes thérapeutiques de sa mise en place (l'étape orthodontique préchirurgicale, l'étape chirurgicale et l'étape orthodontique post-chirurgicale). Une connaissance rigoureuse et un respect des normes contribueront à une procédure chirurgicale moins invasive. chirurgicale, et la biomécanique de traction en fonction de la position de la canine.

\section{- Inspection}

L'inspection s'intéresse aux anomalies de position des dents à proximité de la canine incluse. Le signe de Quintero [1, 9], décrit en 1936, est un signe pathognomonique de l'inclusion canine. La couronne de l'incisive latérale sera déplacée dans le sens opposé à la pression exercée sur sa racine par la canine incluse (fig. 1).

Pour Bassigny (1990) [10-12], toute vestibuloversion accentuée de l'incisive latérale doit faire suspecter une position vestibulaire de la 
canine incluse. En revanche, une distoversion sévère de l'incisive latérale est le signe d'une inclusion canine soit palatine, soit sur la ligne de l'arcade.

\section{- Palpation}

La palpation de la région alvéolaire peut mettre en évidence une voussure anormale de la fibromuqueuse, dure, indolore et non dépressible (fig. 2 et 3) [11].

Si la palpation est négative, un examen radiographique sera toujours nécessaire [8-10].

Dans une étude réalisée par Smailiene en 2002 sur 42 canines incluses, seulement $64 \%$ ont pu être palpées. Ceci a été expliqué par leur situation haute sur le plan vertical $[10,12,13]$.
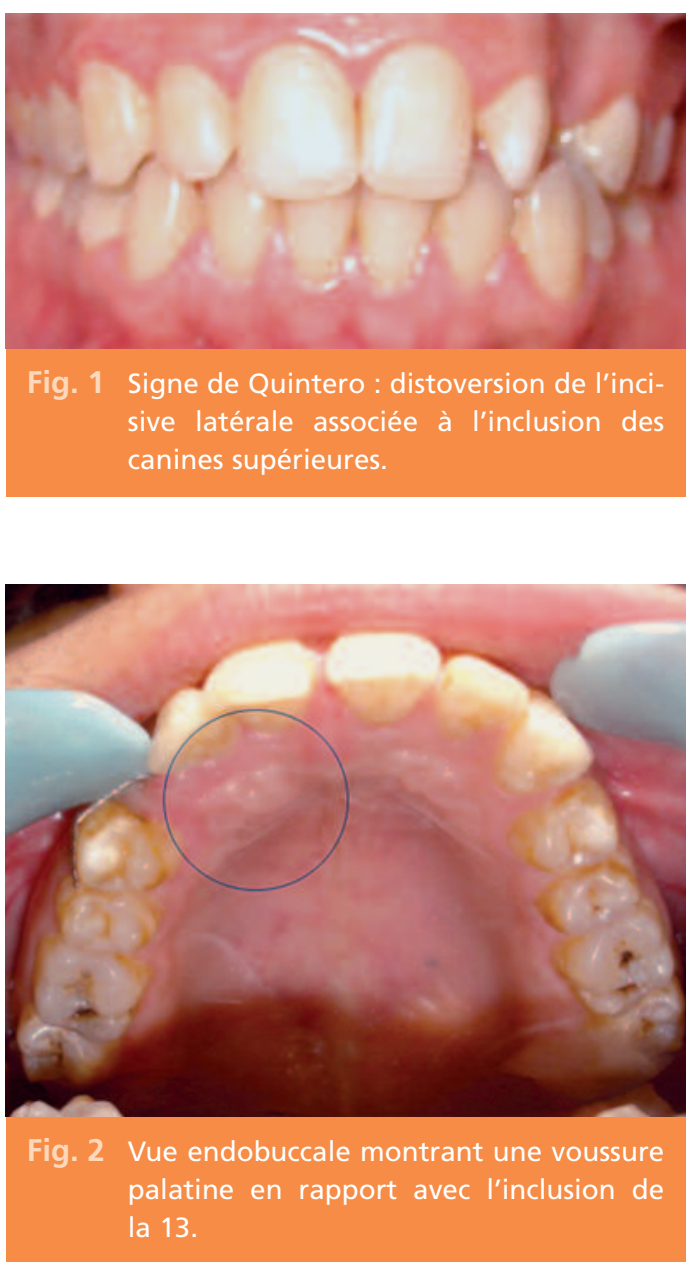

\section{> Moyens radiologiques}

L'examen radiologique est destiné à évaluer avec précision la position, en particulier l'axe des canines par rapport au plan médian et sagittal. En effet, celui-ci représente le facteur décisif en matière de pronostic. La présence d'apex en forme de crochet, ou d'obstacles à l'éruption, est considérée comme étant un indice de pronostic défavorable lors de la mise en place de la dent. Différents clichés radiographiques peuvent être demandés.

\section{- Orthopantomogramme}

II ne permet pas une évaluation précise de la position de la dent incluse dans les trois sens de l'espace.

Toutefois, différents auteurs ont établi des observations pouvant aider le clinicien à localiser la canine incluse dans le sens vestibulopalatin : Mattila et Wolf (1979) [10], Fox et al. (1995), Kuftinec et Shapira (1995) [6], et Mason et al. (2001) [14].

Pour Ericson et Kurol [1, 5, 9, 10, 15-17], le pronostic d'une mise en place sur l'arcade dépend d'un angle formé par l'intersection de l'axe longitudinal (LA) de la dent incluse et l'axe

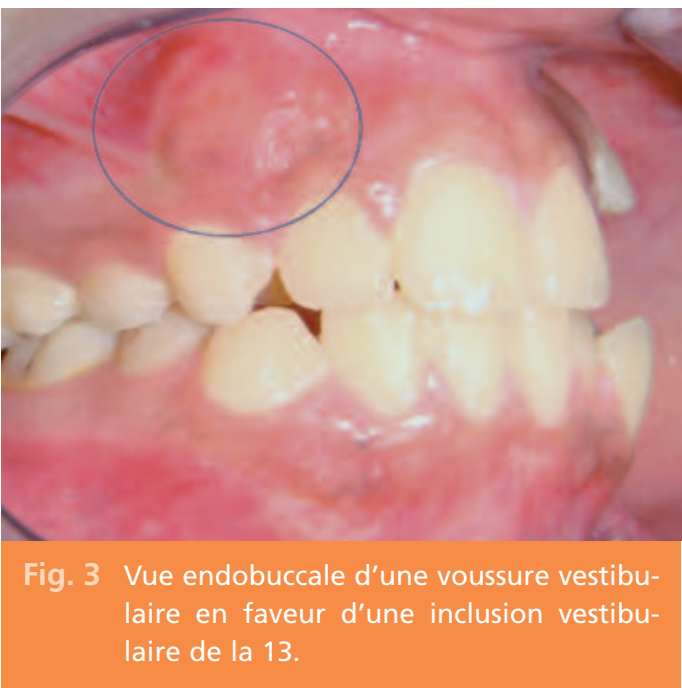




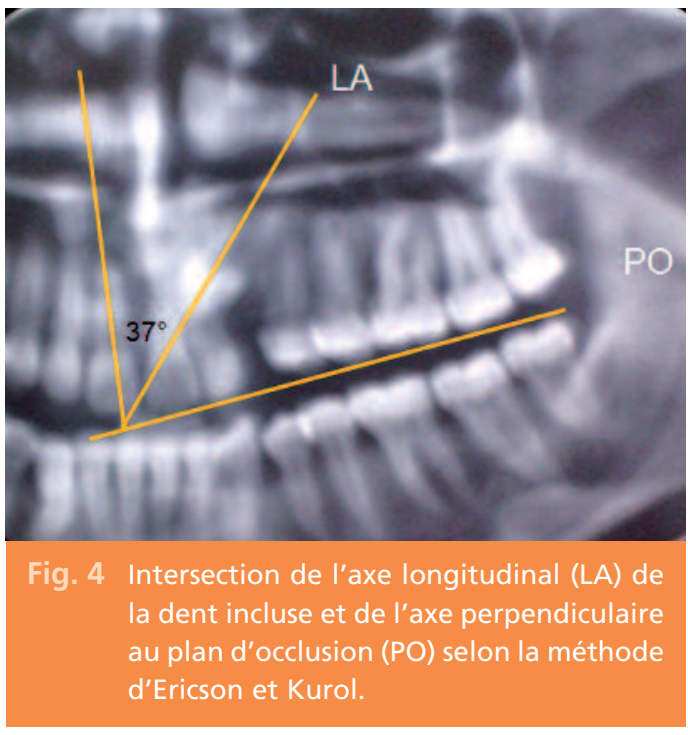

perpendiculaire au plan d'occlusion (PO) à l'emplacement idéal de la canine. Pour les canines inclinées en direction mésiale, le pronostic est favorable lorsque cet angle est inférieur ou égal à $30^{\circ}$. Lorsque la canine est inclinée en direction distale, cet angle devrait être inférieur ou égal à $45^{\circ}$ pour que le pronostic puisse être considéré comme favorable (fig. 4).

La même constatation est faite par Smailiene en 2002 [10, 13, 18], en utilisant une modification de la méthode d'Ericson et Kurol. Il s'agit de repérer la distance entre la canine incluse et la ligne médiane. Pour ce, trois lignes sont dessinées : une tangente distale, l'axe longitudinal et une tangente mésiale à l'incisive latérale. Ces trois lignes objectivent quatre secteurs horizontaux : I, II, III et IV (fig. 5). Une canine incluse en situation médiale par rapport au secteur horizontal III est en position palatine.

Si la dent n'apparaît pas sur le cliché, il faut procéder à d'autres examens pour se prononcer sur son agénésie ou non puisqu'il y a une possibilité que la canine incluse soit située en dehors de la zone tomographiée.

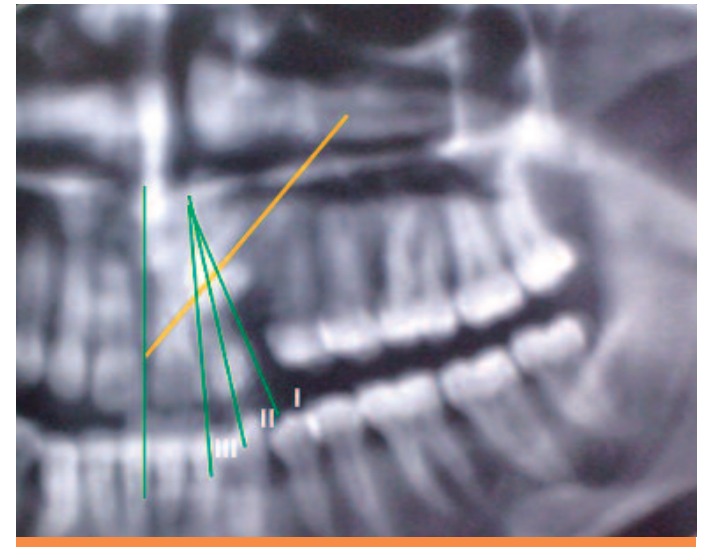

Fig. 5 Les différents secteurs horizontaux selon la méthode d'Ericson et Kurol modifiée par Smailiene.

\section{- Téléradiographie de profil}

Cet examen, réalisé systématiquement lors d'une prise en charge orthodontique, fournit des renseignements dans le plan vertical et dans le plan antéropostérieur. II permet de

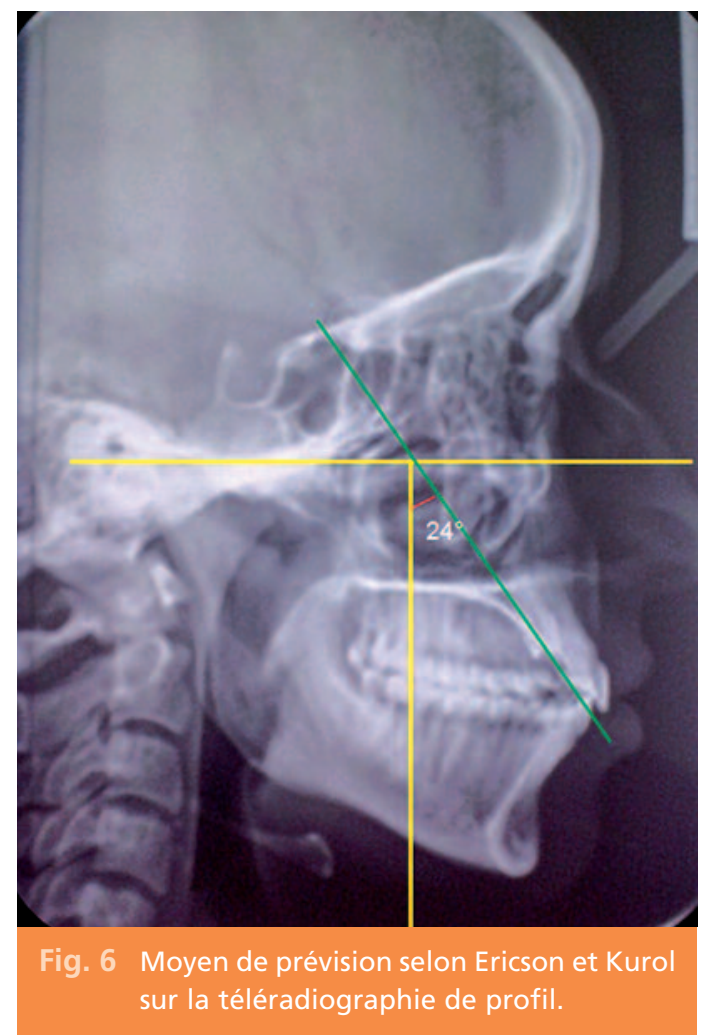




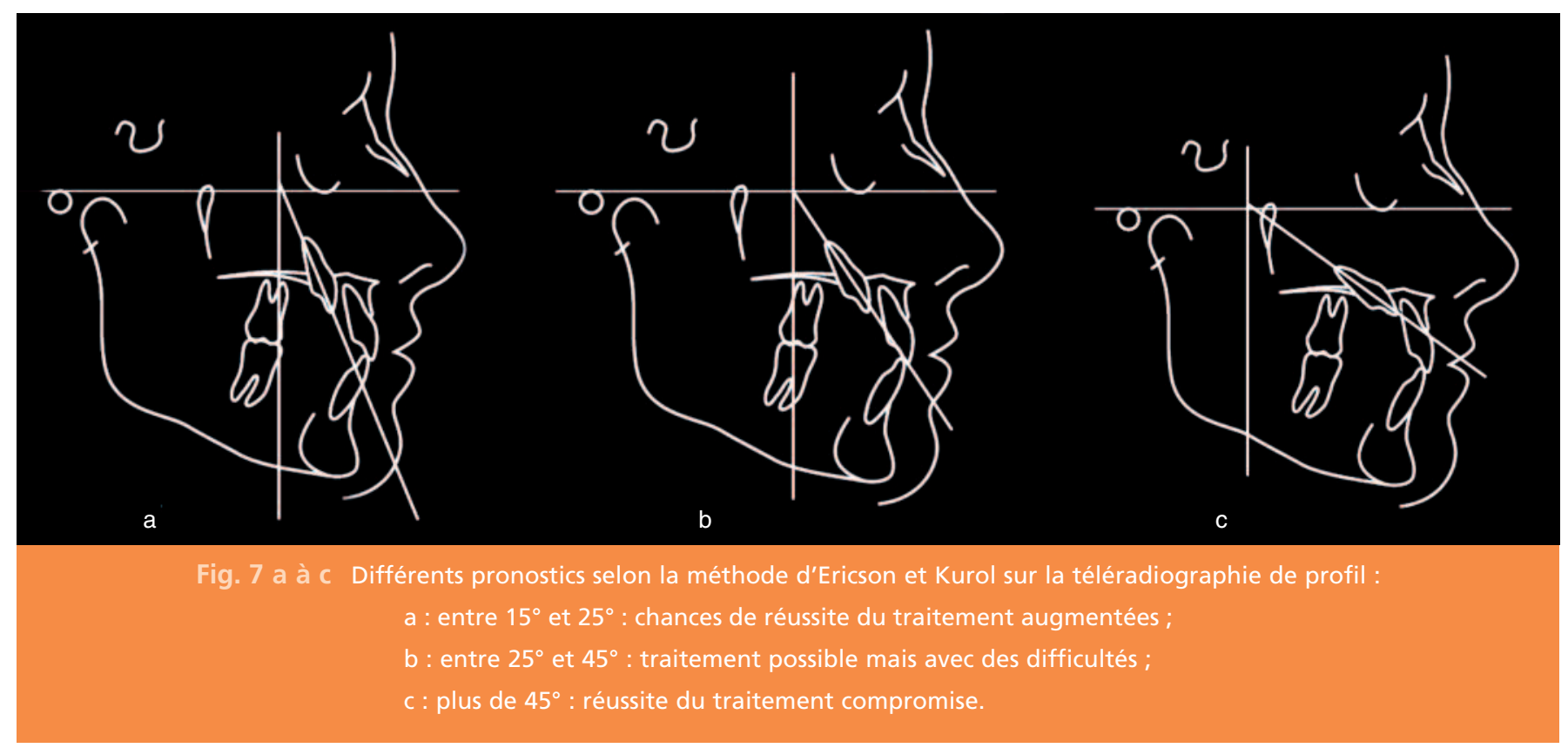

déterminer dans le plan sagittal l'inclinaison de la canine par rapport au plan palatin (PE). Lorsque l'angle formé par l'intersection de l'axe longitudinal de la canine (LA) et le plan palatin (PE) est inférieur à $45^{\circ}$, le pronostic pour la mise en place dans l'arcade doit être considéré comme étant défavorable (Ericson et Kurol) (fig. 6 et 7) [9, 10, 14, 16].

\section{- Clichés occlusaux}

Ces radiographies intrabuccales sont d'une plus grande dimension que les clichés rétroalvéolaires.

Nous les positionnons au niveau du plan d'occlusion. Plusieurs incidences peuvent ainsi être réalisées mais les plus classiques sont :

- la méthode ortho-occlusale de Simpson [19] où le rayon incident est sensiblement perpendiculaire au plan du film. Il permet ainsi de définir la position de la dent incluse par rapport au plan d'occlusion de l'arcade ;

- la méthode dysocclusale proposée en 1907 par Belot $[1,16,17,19]$. Elle s'avère plus intéressante car elle déforme moins les structures dentaires et peut être légèrement latéralisée (fig. 8).

\section{- Scanner}

Le recours à cet examen permet de préciser la position des canines incluses dans les trois dimensions (fig. 9). Couplé à des logiciels de visualisation en 3D, il permet de définir exactement la position, l'axe des dents et leur environnement. II existe aussi des logiciels capables

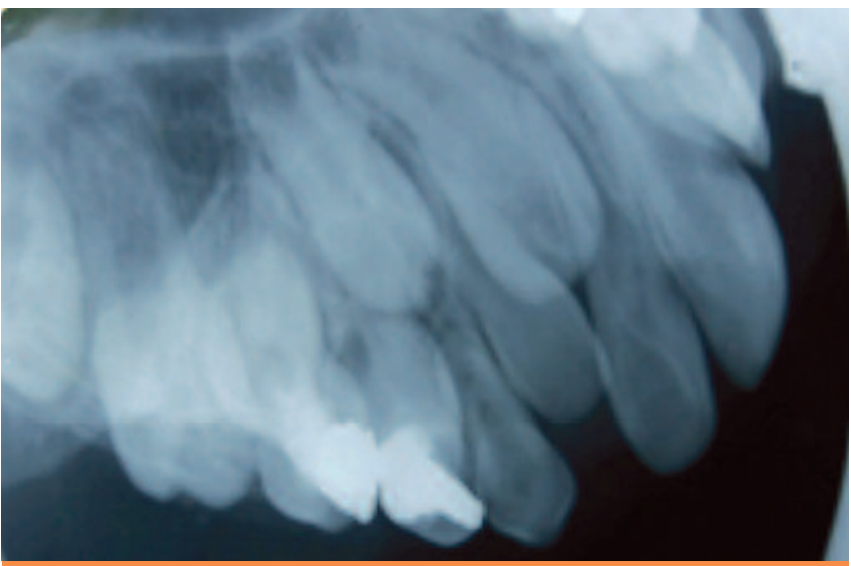

Fig. 8 Cliché occlusal pris selon l'incidence dysocclusale de Belot objectivant l'inclusion de la canine. 

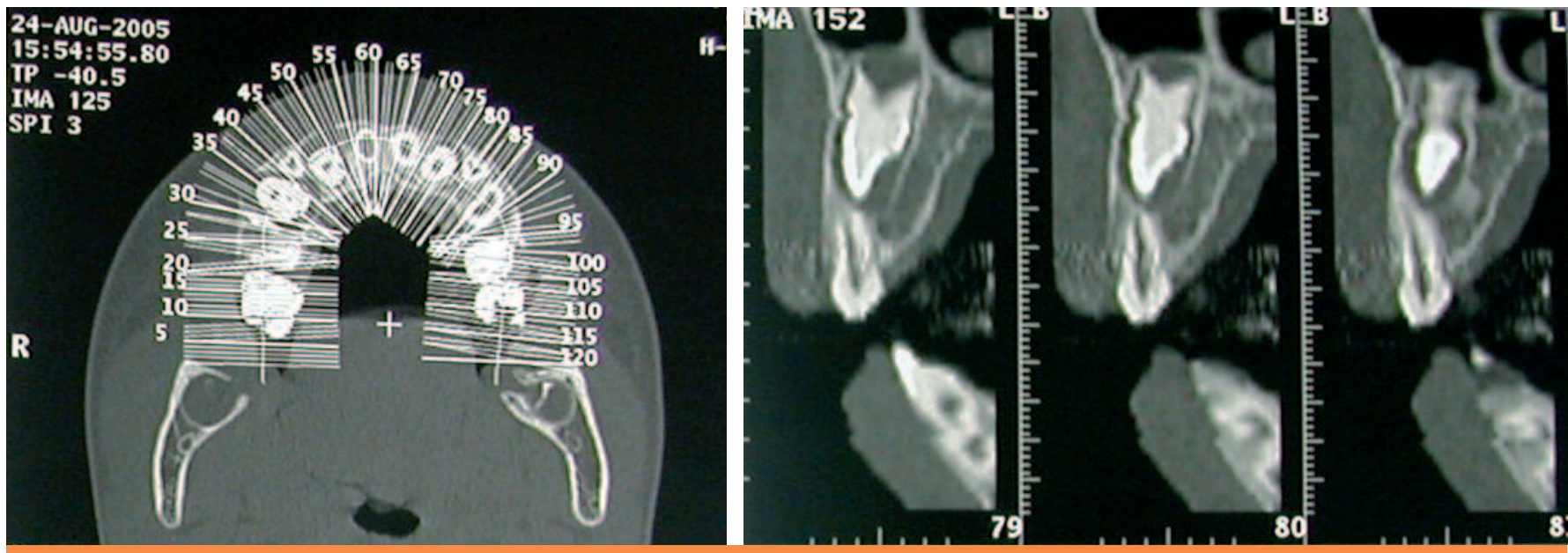

Fig. 9 a et b Différentes coupes tomodensitométriques démontrent la position exacte de la canine incluse.

de transformer ces images tridimensionnelles en des modèles dits "stéréolithographiques" en résine qui représentent la réalité anatomique $[16,20,21]$.

Ceci est très utile pour un abord chirurgical, ainsi que pour l'orientation des tractions, les phases orthodontiques de la désinclusion.

\section{- Cone-beam}

Il fournit des renseignements précieux sur les canines afin de mieux comprendre et traiter ces cas aussi bien chirurgicalement que par l'orthodontie.

Ainsi, on a une idée précise sur la localisation de la canine incluse dans les trois plans de l'espace, la taille du follicule, la quantité de l'os recouvrant la canine, la proximité des dents adjacentes (jusqu'à une distance de 0,5 mm), la présence de résorptions et l'état des structures adjacentes, ainsi que le stade de développement de la canine. II présente l'avantage de nécessiter une dose relativement basse d'irradiation : 50,2 $\mu$ Sv pour les deux maxillaires. Pour le scanner, la dose de radiation absorbée est de l'ordre de 17,6 à 656,9 $\mu$ Sv pour le maxillaire et de 124,9 à $28,4 \mu \mathrm{Sv}$ pour la mandibule [4].

\section{Thérapeutiques}

La mise en place d'une canine incluse doit se faire en trois temps. Un premier temps orthodontique préchirurgical de réouverture et préparation du futur site de mise en place de la canine, puis un second temps chirurgical avec le dégagement de la couronne pour y coller un moyen de traction et enfin un troisième temps orthodontique pour la traction elle-même et la mise sur arcade de la canine.

\section{> Étape orthodontique préchirurgicale}

L'objectif premier de cette phase consiste à aménager le futur site de la canine incluse en lui réservant et en lui créant une place suffisante à son repositionnement, et à réaliser une unité d'ancrage au niveau de l'arcade.

Deux situations se présentent classiquement :

- soit l'aménagement de la place nécessaire n'exige aucune extraction de dent permanente. On peut assister à une éruption spontanée de la canine (fig. 10) ;

- soit cette préparation impose des extractions de dents permanentes. Ce geste devrait 


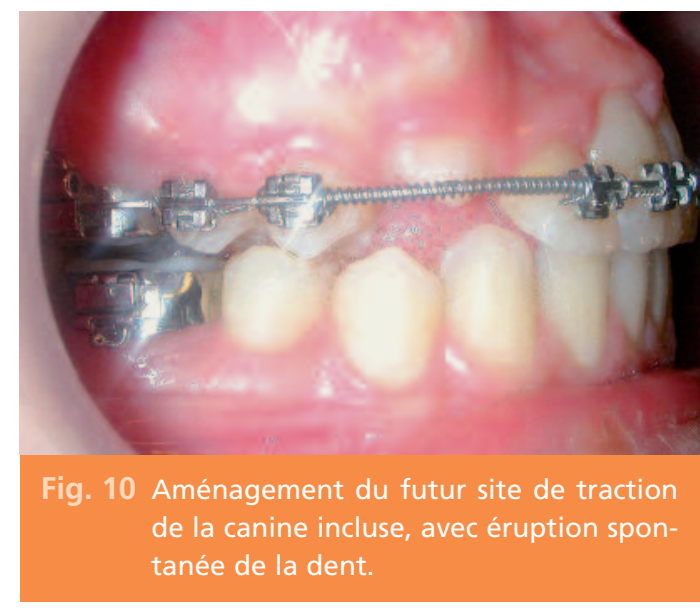

être précédé par un test de traction $[12,22$, 23].

Le test de traction est un bon moyen d'évaluation du risque d'ankylose qui peut pallier les insuffisances des moyens radiologiques.

\section{> Étape chirurgicale}

\section{- Abord chirurgical}

Une bonne prise de conscience parodontale au moment de cette phase permet, dans la majorité des cas cliniques, d'assurer l'intégration de la dent incluse dans un environnement parodontal sain, et d'éviter toute réintervention ultérieure.

Le dégagement chirurgical coronaire devra se faire à minima que ce soit au niveau osseux ou

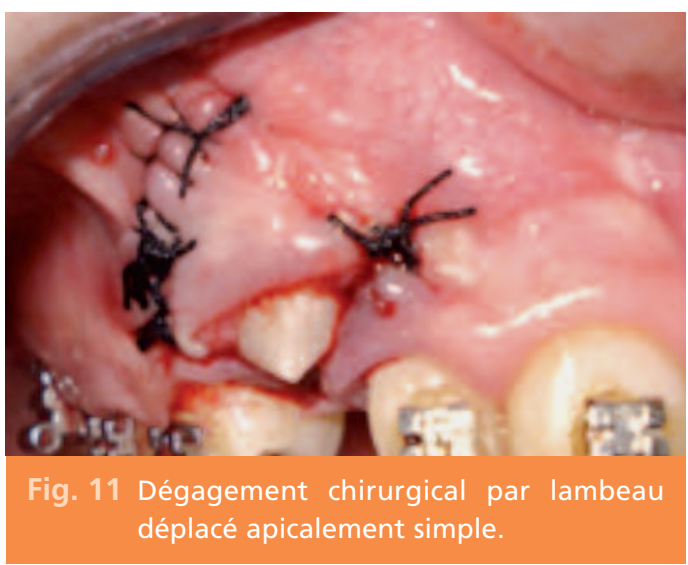

au niveau muqueux, afin de conserver le complexe parodontal du site de traction.

Les auteurs s'accordent pour reconnaître que les problèmes parodontaux sont rares si la canine est palatine.

Classiquement, un lambeau palatin, suivant le collet des dents et d'étendue variable, est réalisé et suivi du décollement de la fibromuqueuse palatine. Puis la crypte osseuse sera soigneusement repérée et aménagée de façon à coller un système de traction. On placera ce système de traction de préférence sur la face vestibulaire afin de réduire toute rotation de la canine. Le lambeau est ensuite repositionné $[22,23]$.

Si la canine est vestibulaire, on veillera à ne pas engendrer un site d'éruption au-dessus de la ligne muccogingivale. Trois types d'intervention peuvent être adoptés :

- le lambeau de translation apicale simple, s'adressant à des canines vestibulaires à grand axe proche de la normale (fig. 11) ;

- le lambeau de translation latérale et apicale, qui trouve son indication dans le cas de canines à axe beaucoup plus oblique et dont le site éruptif présumé est éloigné du couloir éruptif normal ;

- le lambeau repositionné, qui s'adresse aux inclusions très apicales (fig. 12).

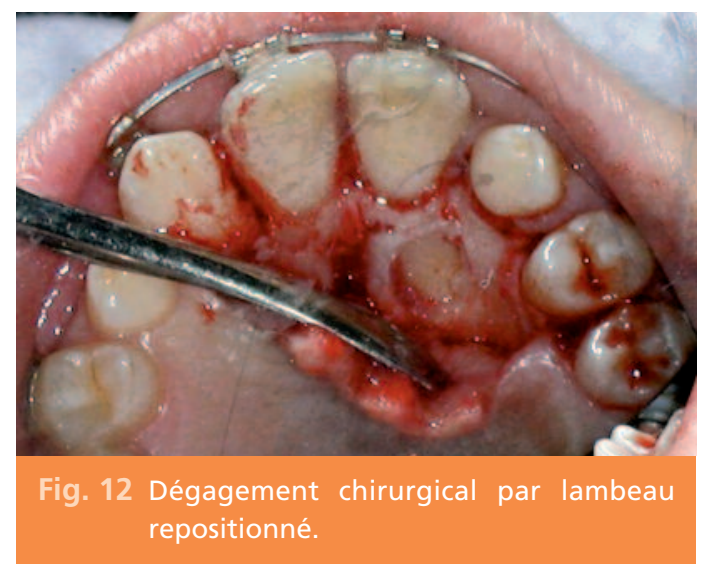


Ces lambeaux consistent en des lambeaux d'épaisseur totale en regard de la gencive adhérente puis d'épaisseur partielle au niveau du pédicule conjonctif $[12,24]$.

\section{- Collage du moyen d'ancrage}

Lors du collage du moyen d'ancrage, il sera très important de respecter certaines étapes afin d'éviter les décollements en cours de traction [25] : la préparation de la surface amélaire, I'hémostase, le mordançage de l'émail, la mise en place de l'attache.

\section{- Préparation de la surface amélaire}

Il s'agit d'effectuer un nettoyage ponctuel à I'alcool.

\section{- Hémostase}

C'est une condition nécessaire à la réalisation d'un bon collage. Toute pollution de la surface de l'émail, par contamination sanguine ou par suintement des bords de la plaie, diminue le pouvoir d'adhésion à l'émail, rendant le collage de mauvaise qualité, voir inefficace.

Pour assurer une bonne hémostase lors du collage, il faut localiser l'origine du saignement :

- le lambeau : il peut saigner, l'aspiration chirurgicale est inopérante car trop ponctuelle, I'hémostase est obtenue grâce à du collagène en poudre ;

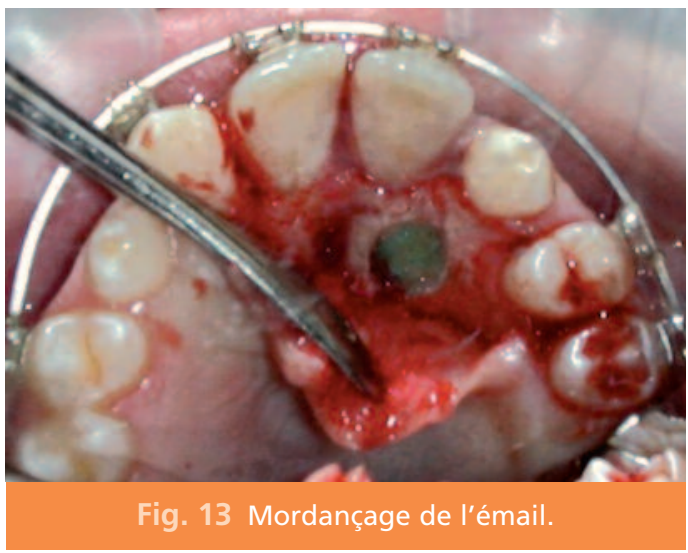

- le sac folliculaire : il est très irrigué et toute hémostase de surface est inopérante, seule une compression permet de conserver un champ opératoire exsangue [23].

\section{- Mordançage de l'émail}

Un gel orthophosphorique est déposé à l'aide d'une seringue munie d'une aiguille fine.

Après 50 secondes, le gel est aspiré puis un rinçage abondant est effectué.

Le séchage est réalisé de préférence par aspiration plutôt que par jet d'air, pour éviter la pollution de surface (fig. 13) [12, 23].

Lors du collage, il sera très important de bien gratter ou polir la zone de collage et de bien sécher avant d'effectuer l'etching car la cuticule Nasmith résiste aux acides et ne permettra pas une résistance suffisante du collage lors de la traction [24].

\section{- Mise en place de l'attache}

Le collage de l'attache peut être effectué par un composite de collage auto- ou photo-polymérisable. Une fois le collage effectué, la solidité de l'ancrage doit être testée afin d'être compatible avec les forces de traction qui seront exercées (fig. 14). Une fenêtre muqueuse pourrait être réalisée afin de permettre un collage différé et éviter l'obstacle muqueux lors de la traction.

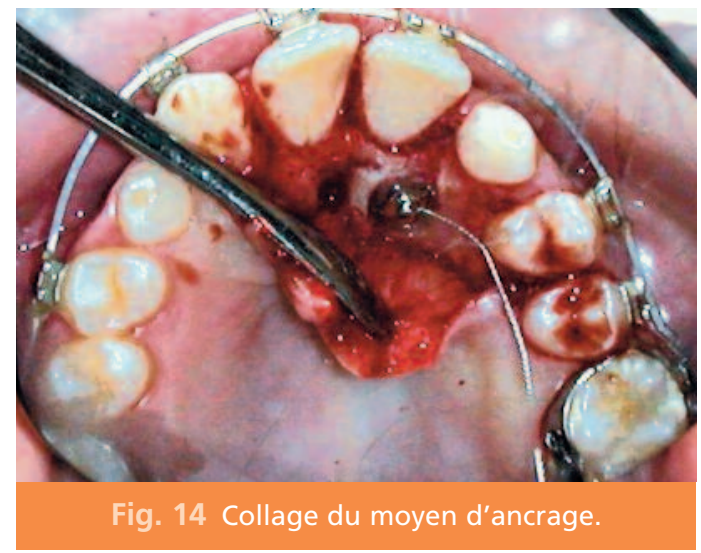




\section{$>$ Étape orthodontique post-chirurgicale}

La mise en place de la canine incluse se fera essentiellement à l'aide de forces très douces, de l'ordre de 30 à 50 grammes. Des forces d'égression trop importantes n'autorisent pas un bon suivi des tissus parodontaux osseux et mucogingivaux. La dent incluse est extrudée avec un parodonte affaibli $[12,24,25]$.

L'attitude thérapeutique la plus adoptée est la traction par appareillage multi-attaches : les dents d'ancrage sont reliées par un arc rigide de forte section, sur lequel est placé un système auxiliaire (traction par élastique, chaînette élastomérique). L'arc principal peut servir également comme générateur de force par sa déformation élastique (fig. 15).

La direction de la traction devra être étudiée afin d'éviter toute gêne au déplacement de la couronne. Si la canine est palatine, elle devra être tractée verticalement et distalement du côté palatin pour ensuite la vestibuler après

\section{Discussion}

La canine est une des dents les plus importantes de l'arcade dentaire. Elle joue un rôle à la fois esthétique et fonctionnel.

Le pronostic de la thérapeutique orthodontico-chirurgicale varie selon différents critères. Il semble que les canines, dont l'inclusion est secondaire à une fermeture d'espace ou à un obstacle tel que la présence d'un germe surnuméraire, sont d'excellent pronostic.

En cas de présence de kyste, le pronostic est variable, suivant l'importance de ce dernier, les gros kystes entraînent en général de fortes malpositions, difficiles à rattraper. son éruption. Une traction trop vestibulaire dès le début risque de ralentir le déplacement et de toucher les racines voisines.

Dans ce type de configuration, la canine se bloque, n'évolue plus ou même peut résorber la racine qui gêne son déplacement.

Si la canine est vestibulaire, la traction devra être progressive en évitant de faire sortir sa couronne dans la gencive libre, au-dessus de la ligne muccogingivale [23-25].

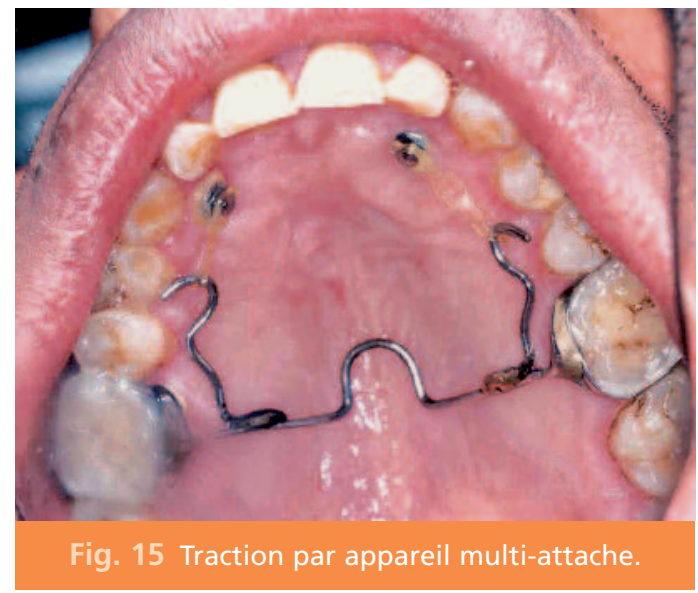

On peut aussi affirmer que les cas les plus favorables sont les cas ou la dent n'est pas très éloignée de son site d'éruption avec une direction assez voisine de la normale. Bishara affirme qu'une dent incluse en position horizontale est de mauvais pronostic $[1-3,10]$.

Le pronostic varie également avec l'âge : plus l'âge du patient est jeune, plus le pronostic est bon.

Ceci est confirmé par une étude réalisée par A. Becker et al. sur deux groupes d'âges différents ayant subi une désinclusion de canine incluse maxillaire de difficulté semblable. 


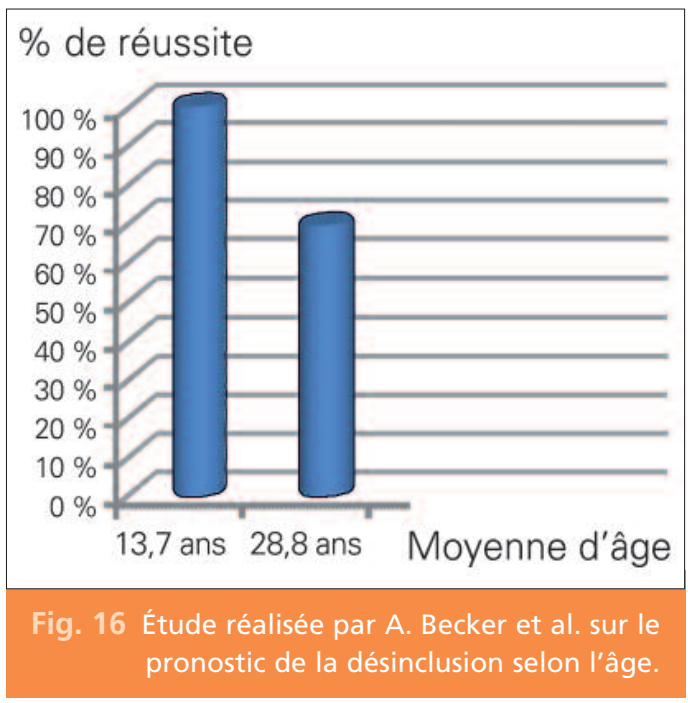

Ils ont conclu que le pronostic chez les patients jeunes est plus élevé [1] (fig. 16).

Malheureusement, la thérapeutique orthodontico-chirurgicale peut aboutir à l'échec. Pour cela, il est indispensable d'informer les patients et leur entourage de cette possibilité, même si elle est minime.

\section{Conclusion}

La mise en place d'une canine incluse est un acte simple, mais il convient $d$ 'avertir le patient de la durée du traitement et des risques d'échecs.

L'évolution de l'imagerie, des techniques thérapeutiques, des matériaux et la prise en compte de l'environnement parodontal ont
La spécificité de l'échec de la mise en place des dents incluses est qu'il peut intéresser la dent incluse elle-même (ankylose, coudure radiculaire, récession radiculaire, perte de gencive attachée, rhizalyse), comme il peut intéresser les dents adjacentes (résorption, mouvements parasites), sans oublier bien sûr l'échec au niveau du patient $[10,17,22,23]$.

En somme, il convient de relever que les méthodes conventionnelles de diagnostic ne permettent pas seulement de déterminer la position des canines incluses, mais qu'elles sont également susceptibles de fournir des informations concernant le pronostic.

Quant au problème souvent évoqué du risque de lésions des dents voisines, la tomographie numérique est actuellement le moyen de diagnostic de choix, de même que cette technique permet de définir la planification des forces mécaniques nécessaires dans les cas de positions ectopiques défavorables et lors des étapes initiales du traitement [6-8].

permis de faciliter la traction de la canine incluse.

Cet acte pluridisciplinaire offre aujourd'hui un pronostic favorable de mise en place esthétique et fonctionnelle, à condition de respecter rigoureusement les conditions de réussite du diagnostic à la thérapeutique.

\section{Bibliographie}

1. Chambas $\mathrm{C}$. Désinclusion et mise en place des dents retenues.

Encycl Méd Chir (Elsevier SAS,
Paris), Odontologie,

1997;23-492-A-10. 
2. Torres-Lagares $D$,

Lesclous $\mathrm{P}$,

Garcia-Calderon M,

Gutierrez JL.

Complications de l'inclusion de la canine maxillaires : cas cliniques et revue de la littérature. Clinic 2007;28:699-705.

3. Pajoni D, Korbendau J-M, Le Bras C. Inclusion profonde des canines maxillaires : un danger pour les prémolaires. Orthod Fr 2003;74:29-35.

4. Walker I, Enciso R, Mah J. Three dimensional localization of maxillary canines with cone-beam computed tomography. Am J Orthod Dentofacial Orthop 2005;128:418-23.

5. Guérin $\mathrm{T}$.

Apport de l'imagerie au diagnostic topographique des canines maxillaires incluses.

Act Odonto Stomatol 1999;205:27-41.

6. Kuftinec MM, Shapira Y. The impacted maxillary canines:

I. Review of concepts. II. Clinical approaches and solutions. ASDC J Dent Child 1995;62:317-34.

7. Guiral H, Medina L, Cavezian R, Pasquet G. Localisation des canines incluses : mise au point. Act Odonto Stomatol 2009;245:63-70.

8. Crismani AG,

Freudenthaler JW, Weber R, Bantleon HP.

Canines supérieures incluses - méthodes conventionnelles de diagnostic radiologique et de traitement.

Rev Mens Suisse

Odontostomatol

2000;110:1264-7.

9. Richardson G, Russell KA.

A review of impacted permanent maxillary cuspids: diagnosis and prevention. J Can Dent Assoc 2000;66(9):497-501.

10. Maverna R, Gracco A. Different diagnostic tools for the localization of impacted maxillary canines: clinical considerations. Prog Orthod 2007;8(1):28-44.

11. Bassigny $F$. Les signes prémonitoires d'inclusion des canines supérieures : une approche préventive. Rev Orthop Dento Faciale 1990;24:91-102.

12. Delsol L, Orti V, Chouvin M, Canal P.

Canines et incisives maxillaires incluses : diagnostic et thérapeutique. Encycl Méd Chir (Elsevier SAS, Paris), Odontologie/Orthopédie dentofaciale 2006;23-492-A-11.

13. Smailiene D. Localization of impacted maxillary canines by palpation and orthopantomography. Medicina (Kaunas) 2002:38:825-9.

14. Mason C, Papadakou P, Roberts GJ.

The radiographic localization of impacted maxillary canines: a comparaison of methods. Eur J Orthod 2001,23(1):25-34
15. Ericson S, Kurol J. Radiographic examination of ectopically erupting maxillary canines. Am J Orthod Dentofacial Orthop 1987;91(6):483-92.

16. Teman G, Lacan A, Suissa M Sarazin L.

Stratégie des explorations en imagerie maxillofaciale. Guidelines for prescribing dental radiography.

Encycl Méd Chir

(Elsevier SAS, Paris), dentisterie 2004;1(3):334-44.

17. Liu DG, Zhang WL, Zhang ZY, Wu YT, Ma XC. Localization of impacted maxillary canines and observation of adjacent incisor resorption with cone-beam computed tomography.

Oral Surg Oral Med Oral Pathol Oral Radiol Endod 2008;105(1):91-8.

18. Chaushu S, Chaushu G, Becker A.

The use of panoramic radiographs to localize displaced maxillary canines. Oral Surg Oral Med Oral Path Oral Radio Endod 1999;88:511-6.

19. Mailland M. Techniques de radiologie dentaire. Paris : Masson, 1987.

20. Sudhakar S, Patil K, Mahima VG.

Localization of impacted permanent maxillary canine using single panoramic radiograph.

Indian J Dent Res 2009;20(3):340-5.

21. Bossard D, N. Dubos F, et al. 3D Computed-assisted surgery in orthodontic treatment of impacted canines in palatal position. 
In Lemke HU, et al. editors.

\section{CARS 2004.}

Computer Assisted

Radiology and Surgery.

Proceedings of

the 18th International

Congress and Exhibition,

Chicago, USA,

June 23-26, 2004.

International Congress

Series 2004;1268:1203-8.

22. Tausche E, Harzer W. Treatment of a patient with Class II malocclusion, impacted maxillary canine with a dilacerated root, and peg-shaped lateral incisors.

Am J Orthod Dentofacial Orthop 2008;133(5):762-7.

23. Kokich VG.

Surgical and

orthodontic management

of impacted

maxillary canines.

Am J Orthod Dentofacial

Orthop 2004;126(3):278-83.

24. Schmidt $A D$, Kokich VG.

Periodontal response to early uncovering, autonomous eruption, and orthodontic alignment of palatally impacted maxillary canines. Am J Orthod Dentofacial Orthop 2007;131(4):449-55.

25. Zuccati G, Ghobadlu J, Nieri M, Clauser C. Factors associated with the duration of forced eruption of impacted maxillary canines: a retrospective study. Am J Orthod Dentofacial Orthop 2006;130(3):349-56.

\section{SUMMARY}

\section{Orthodontico-surgical traction}

of impacted canines: means of failures forecasts

Mohamed Fadel ZEROUAOUI

Loubna BAHIJE

Fatima ZAOUI

Wiam RERHRHAYE

\section{Keywords \\ - impacted canine - orthodontic-surgical traction \\ - failures}

Each tooth has its importance in the oral cavity and therefore its absence could cause an imbalance and have an impact on the health of surrounding oral structures. When dental impaction occurs, clinical and radiological examinations are necessary for both diagnosis and prognosis. Thus, several therapeutic options are possible ranging from abstention to surgical extractions using alveolectomy. However, the treatment most widely adopted and most desired is the establishment of orthodontico-surgical traction. Our work proposes to put the point on the means of failures forecasts which this therapeutic can meet.

\section{Questions-Réponses}

$>1$. - Le principe de l'orthodontie pré-chirurgicale des canines incluses est:

a. I'aménagement du futur site de la canine incluse ;

b. le nivellement de l'arcade dentaire ;

c. la réalisation de l'unité d'ancrage au niveau de l'arcade. 
> 2. - Le lambeau de translation latérale et apicale trouve son indication dans les cas:

a. de canines vestibulaires à grand axe proche de la normale ;

b. de canines à axe beaucoup plus oblique et dont le site éruptif présumé est éloignée du couloir éruptif normal.

> 3. - La mise en place de la canine incluse se fera essentiellement à l'aide de:

a. une force d'égression importante ;

b. une force très douce, de l'ordre de 30 à 50 grammes.

$$
q: \varepsilon-q: 乙-\partial \jmath \partial \mathrm{e}:\llcorner
$$

\section{Apprenez à respirer à vos enfants}

\section{Jean-Paul Allaux}

Les maladies ORL avec leur prolongement au niveau pulmonaire sont malheureusement de plus en plus répandues de nos jours et à tout âge.

La pollution atmosphérique, les appartements surchauffés ou enfumés par des parents tabagiques, les rues des villes saturées de gaz d'échappement... tous ces maux contemporains bien connus sont bien souvent responsables de I'installation de ces affections dès la petite enfance. Ils freinent par ailleurs le développement harmonieux du corps en installant parfois des déformations vertébrales mais aussi du palais et des mâchoires provoquant une mauvaise implantation des dents.

Fruit d'une longue expérience, ce livre se veut avant tout pratique. De nombreux exercices spécifiques à faire à la maison ou à l'école, seul ou en groupe, permettront aux éducateurs, parents et enseignants de trouver remède à ces différentes affections et d'aider efficacement les enfants à s'épanouir pleinement.

Un livre pour apprendre à respirer à nos enfants.

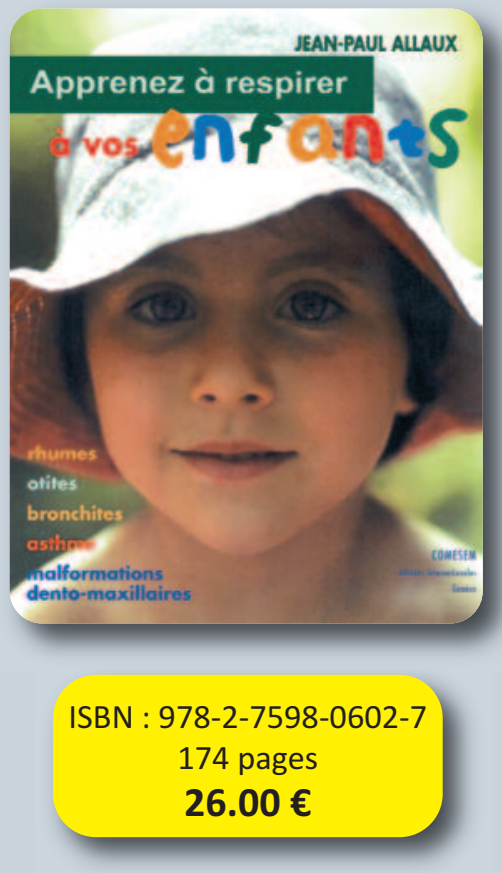

\section{Commandez directement sur : www.librairie-garanciere.com}

